Research Article

\title{
Construction of Type 2 Poly-Changhee Polynomials and Its Applications
}

\author{
Ghulam Muhiuddin $\left(\mathbb{D},{ }^{1}\right.$ Waseem Ahmad Khan $\left(\mathbb{D},{ }^{2}\right.$ and Jihad Younis $\mathbb{D}^{3}$ \\ ${ }^{1}$ Department of Mathematics, University of Tabuk, Tabuk 71491, Saudi Arabia \\ ${ }^{2}$ Department of Mathematics and Natural Sciences, Prince Mohammad Bin Fahd University, P.O. Box 1664, \\ Al Khobar 31952, Saudi Arabia \\ ${ }^{3}$ Department of Mathematics, Aden University, P.O. Box, Khormaksar 6014, Yemen
}

Correspondence should be addressed to Jihad Younis; jihadalsaqqaf@gmail.com

Received 11 August 2021; Accepted 12 November 2021; Published 10 December 2021

Academic Editor: Yuan Yi

Copyright (C) 2021 Ghulam Muhiuddin et al. This is an open access article distributed under the Creative Commons Attribution License, which permits unrestricted use, distribution, and reproduction in any medium, provided the original work is properly cited.

In this paper, we introduce type 2 poly-Changhee polynomials by using the polyexponential function. We derive some explicit expressions and identities for these polynomials, and we also prove some relationships between poly-Changhee polynomials and Stirling numbers of the first and second kind. Also, we introduce the unipoly-Changhee polynomials by employing unipoly function and give multifarious properties. Furthermore, we provide a correlation between the unipoly-Changhee polynomials and the classical Changhee polynomials.

\section{Introduction}

Special polynomials and their generating functions have vital roles in several branches of arithmetic, probability, statistics, mathematical physics, and additionally engineering. Since polynomials are appropriate for applying wellknown operations like by-product and integral, polynomials are helpful to check real-world issues within the said areas. As an example, generating functions for special polynomials with their congruousness properties, repetition relations, process formulae, and regular add involving these polynomials are studied in recent years (see [1-4]).

For $j \geq 0$, the Stirling numbers of the first kind are defined by the following (see $[1,2,5-14])$ :

$$
(\xi)_{j}=\sum_{l=0}^{j} S_{1}(j, l) \xi^{l}
$$

where $(\xi)_{0}=1$ and $(\xi)_{j}=\xi(\xi-1), \ldots,(\xi-j+1),(j \geq 1)$. From (1), it is easy to see that (see $[4,15-22]$ )

$$
\frac{1}{r !}(\log (1+z))^{r}=\sum_{j=r}^{\infty} S_{1}(j, r) \frac{z^{j}}{j !}, \quad(r \geq 0) .
$$

For $j \geq 0$, the Stirling numbers of the second kind are defined by the following (see $[3,11-16])$ :

$$
\xi^{j}=\sum_{l=0}^{j} S_{2}(j, l)(\xi)_{l}
$$

From (3), we see that

$$
\frac{1}{r !}\left(e^{z}-1\right)^{r}=\sum_{j=r}^{\infty} S_{2}(j, r) \frac{z^{j}}{j !} .
$$


The Bernoulli $B_{j}(\xi)$, Euler $E_{j}(\xi)$, and Genocchi $G_{j}(\xi)$ polynomials are defined by the following (see $[1,6,7])$ :

$$
\frac{z}{e^{z}-1} e^{\xi z}=\sum_{j=0}^{\infty} B_{j}(\xi) \frac{z^{j}}{j !}, \quad|z|<2 \pi, \frac{2}{e^{z}+1} e^{\xi z}=\sum_{j=0}^{\infty} E_{j}(\xi) \frac{z^{j}}{j !},|z|<\pi,
$$

$\frac{2 z}{e^{z}+1} e^{\xi z}=\sum_{j=0}^{\infty} G_{j}(\xi) \frac{z^{j}}{j !},|z|<\pi$,

respectively.

Let the Changhee polynomials $C h_{j}(\xi)$ be given by the following (see $[2,13,14,16])$ :

$$
\int_{\mathbb{Z}_{p}}(1+z)^{\xi+\eta} \mathrm{d} \mu_{-1}(\eta)=\frac{2}{2+z}(1+z)^{\xi}=\sum_{j=0}^{\infty} C h_{j}(\xi) \frac{z^{j}}{j !} .
$$

In the case when $\xi=0, C h_{j}=C h_{j}(0)$ are called the Changhee numbers.

Moreover, we have the following (see [13]):

$$
\begin{aligned}
C h_{j}(\xi) & =\sum_{l=0}^{j} E_{l}(\xi) S_{1}(j, l), \\
E_{j}(\xi) & =\sum_{l=0}^{j} C h_{l}(\xi) S_{2}(j, l), \quad(j \geq 0) .
\end{aligned}
$$

The polyexponential function as an inverse to the polylogarithm function is defined by Kim and Kim [12] to be

$$
\operatorname{Ei}_{k}(\xi)=\sum_{j=1}^{\infty} \frac{\xi^{j}}{(j-1) ! j^{k}}, \quad(k \in \mathbb{Z}) .
$$

We note that

$$
\operatorname{Ei}_{1}(\xi)=\sum_{j=1}^{\infty} \frac{\xi^{j}}{j !}=e^{\xi}-1 .
$$

In 2019, Kim and Kim [12] introduced the poly-Bernoulli polynomials which are defined by

$$
\frac{\mathrm{Ei}_{k}(\log (1+z))}{e^{z}-1} e^{\xi z}=\sum_{j=0}^{\infty} B_{j}^{(k)}(\xi) \frac{z^{j}}{j !}
$$

Letting $\xi=0, B_{j}^{(k)}=B_{j}^{(k)}(0)$ are called the poly-Bernoulli numbers.

For $k \in \mathbb{Z}$, the polylogarithm function is defined by the following (see $[5,21])$ :

$$
\operatorname{Li}_{k}(\xi)=\sum_{j=1}^{\infty} \frac{\xi^{j}}{j^{k}},(|\xi|<1) .
$$

Note that

$$
\operatorname{Li}_{1}(\xi)=\sum_{j=1}^{\infty} \frac{\xi^{j}}{j}=-\log (1-\xi) .
$$

Lee et al. [22] introduced the type 2 poly-Euler polynomials which are given by

$$
\frac{\mathrm{Ei}_{k}(1+2 z)}{z\left(e^{z}+1\right)} e^{\xi z}=\sum_{j=0}^{\infty} E_{j}^{(k)}(\xi) \frac{z^{j}}{j !}
$$

In the case when $\xi=0, E_{j}^{(k)}=E_{j}^{(k)}(0)$ are called the type 2 poly-Euler numbers.

The Daehee polynomials are defined by the following (see $[7,16])$ :

$$
\frac{\log (1+z)}{z}(1+z)^{\xi}=\sum_{j=0}^{\infty} D_{j}(\xi) \frac{z^{j}}{j !} .
$$

When $\xi=0, D_{j}=D_{j}(0)$ are called the Daehee numbers.

The following paper is as follows. In Section 2, we introduce type 2 poly-Changhee polynomials and numbers and derive some identities of these polynomials. We derive some recurrence relations and relationships between Bernoulli number, Euler numbers, and Daehee numbers. In Section 3, we introduce unipoly-Changhee polynomials and investigate some identities of these polynomials.

\section{Type 2 Poly-Changhee Numbers and Polynomials}

In this section, we define type 2 poly-Changhee polynomials by using the polyexponential functions and represent the usual Changhee polynomials (more precisely, the values of Changhee polynomials at 1 ) when $k=1$. At the same time, we give explicit expressions and identities involving polynomials.

For $k \in \mathbb{Z}$, we define type 2 poly-Changhee polynomials by means of the following exponential generating function (in a suitable neighborhood of $z=0$ ) including the polyexponential function given as follows:

$$
\frac{\mathrm{Ei}_{k}(\log (1+2 z))}{z(2+z)}(1+z)^{\xi}=\sum_{j=0}^{\infty} C h_{j}^{(k)}(\xi) \frac{z^{j}}{j !} .
$$

At the point $\xi=0, C h_{j}^{(k)}=C h_{j}^{(k)}(0)$ are called type 2 poly-Changhee numbers.

For $k=1$, by using (9) and (16), we see that

$$
\begin{aligned}
\frac{\mathrm{Ei}_{1}(\log (1+2 z))}{z(2+z)}(1+z)^{\xi} & =\frac{2}{2+z}(1+z)^{\xi} \\
& =\sum_{j=0}^{\infty} C h_{j}(\xi) \frac{z^{j}}{j !}, \quad(j \geq 0),
\end{aligned}
$$

where $C h_{j}(\xi)$ are called the Changhee polynomials (see equation (7)).

Theorem 1. Let $j$ be the nonnegative number and $k \in \mathbb{Z}$. Then,

$$
C h_{j}^{(k)}(\xi)=\sum_{l=0}^{j}\left(\begin{array}{l}
j \\
l
\end{array}\right) \sum_{i=0}^{l} \frac{1}{(i+1)^{k-1}} \frac{S_{1}(l+1, i+1) 2^{l}}{l+1} C h_{j-l}(\xi) .
$$

Proof. By (16), we have 


$$
\begin{aligned}
\frac{\operatorname{Ei}_{k}(\log (1+2 z))}{z(2+z)}(1+z)^{\xi} & =\frac{1}{z(2+z)}(1+z)^{\xi} \sum_{i=0}^{\infty} \frac{1}{(i+1)^{k-1}} \sum_{l=i+1}^{\infty} S_{1}(l, i+1) \frac{2^{l} z^{l}}{l !} \\
& =\frac{2}{2+z}(1+z)^{\xi} \sum_{i=0}^{\infty} \frac{1}{(i+1)^{k-1}} \sum_{l=i}^{\infty} \frac{S_{1}(l+1, i+1)}{l+1} \frac{2^{l} z^{l}}{l !} \\
& =\sum_{j=0}^{\infty} C h_{j}(\xi) \frac{z^{j}}{j !} \sum_{i=0}^{\infty} \frac{1}{(i+1)^{k-1}} \sum_{l=i}^{\infty} \frac{S_{1}(l+1, i+1) 2^{l}}{l+1} \frac{z^{l}}{l !} \\
& =\sum_{j=0}^{\infty}\left(\sum_{l=0}^{j}\left(\begin{array}{l}
j \\
l
\end{array}\right) \sum_{i=0}^{l} \frac{1}{(i+1)^{k-1}} \frac{S_{1}(l+1, i+1) 2^{l}}{l+1} C h_{j-l}(\xi)\right) \frac{z^{j}}{j !} .
\end{aligned}
$$

In view of (16) and (19), we get (17).

Corollary 1. Let $j$ be the nonnegative number. Then,

$$
C h_{j}(\xi)=\sum_{l=0}^{j}\left(\begin{array}{l}
j \\
l
\end{array}\right) \sum_{i=0}^{l} \frac{S_{1}(l+1, i+1) 2^{l}}{l+1} C h_{j-l}(\xi) .
$$

The higher-order Bernoulli polynomials are defined by the following (see [11]):

$$
\left(\frac{z}{\log (1+z)}\right)^{r}(1+z)^{\xi-1}=\sum_{j=0}^{\infty} B_{j}^{(j-r+1)}(\xi) \frac{z^{j}}{j !}, \quad(r \in \mathbb{C}),
$$

where $B_{j}^{(r)}(\xi)$ are the Bernoulli polynomials of order $\alpha$ by

$$
\left(\frac{z}{e^{z}-1}\right)^{r} e^{\xi z}=\sum_{j=0}^{\infty} B_{j}^{(r)}(\xi) \frac{z^{j}}{j !} .
$$

Theorem 2. Let $j$ be the nonnegative number. Then,

$$
\begin{aligned}
C h_{j}^{(k)}= & \sum_{l=0}^{j}\left(\begin{array}{l}
j \\
l
\end{array}\right) \sum_{l=0}^{\infty} \\
& 2^{l} \times \sum_{l_{1}+\ldots+l_{k-1}=l}\left(\begin{array}{c}
l \\
l_{1}, \ldots, l_{k-1}
\end{array}\right) \frac{B_{l_{1}}^{\left(l_{1}\right)}}{l_{1}+1} \frac{B_{l_{2}}^{\left(l_{2}\right)}}{l_{1}+l_{2}+1} \cdots \frac{B_{l_{k-1}}^{\left(l_{k-1}\right)}}{l_{1}+\cdots+l_{k-1}+1} C h_{j-l} .
\end{aligned}
$$

Proof. From (4), we have

$$
\begin{aligned}
\frac{d}{\mathrm{~d} \xi} \mathrm{Ei}_{k}(\log (1+2 \xi)) & =\frac{d}{\mathrm{~d} \xi} \sum_{j=1}^{\infty} \frac{(\log (1+2 \xi))^{j}}{(j-1) ! j^{k}} \\
& =\frac{2}{1+2 \xi} \sum_{j=1}^{\infty} \frac{j(\log (1+2 \xi))^{j-1}}{(j-1) ! j^{k}}
\end{aligned}
$$

$$
\begin{aligned}
& =\frac{2}{(1+2 \xi) \log (1+2 \xi)} \sum_{j=1}^{\infty} \frac{(\log (1+2 \xi))^{j}}{(j-1) ! j^{k-1}} \\
& =\frac{2}{(1+2 \xi) \log (1+2 \xi)} \operatorname{Ei}_{k-1}(\log (1+2 \xi)) .
\end{aligned}
$$

For $k \geq 1$, using equations (21) and (24), we find 


$$
\begin{aligned}
\operatorname{Ei}_{k}(\log (1+2 \xi))= & \int_{0}^{\xi} \frac{1}{(1+2 z) \log (1+2 z)} \\
& \times \int_{0}^{z} \frac{1}{(1+2 z) \log (1+2 z)} \cdots \int_{0}^{z} \frac{z}{(1+2 z) \log (1+2 z)} \mathrm{d} z \mathrm{~d} z, \ldots, \mathrm{d} z . \\
= & 2 \xi \sum_{l=0}^{\infty} 2^{l} \sum_{l_{1}+\cdots+l_{k-1}=l}\left(l_{1}, \ldots, l_{k-1}\right) \frac{B_{l_{1}}^{\left(l_{1}\right)}}{l_{1}+1} \frac{B_{l_{2}}^{\left(l_{2}\right)}}{l_{1}+l_{2}+1} \cdots \frac{B_{l_{k-1}}^{\left(l_{k-1}\right)}}{l_{1}+\cdots+l_{k-1}+1} \frac{\xi^{l}}{l !} .
\end{aligned}
$$

From (25), we observe that

$$
\begin{aligned}
\sum_{j=0}^{\infty} C h_{j}^{(k)} \frac{\xi^{j}}{j !}= & \frac{1}{\xi(\xi+2)} \operatorname{Ei}_{k}(\log (1+2 \xi)) \\
= & \frac{2 \xi}{\xi(\xi+2)} \\
& \times \sum_{l=0}^{\infty} 2^{l} \sum_{l_{1}+\cdots+l_{k-1}=l}\left(l_{1}, \ldots, l_{k-1}\right) \frac{B_{l_{1}}^{\left(l_{1}\right)}}{l_{1}+1} \frac{B_{l_{2}}^{\left(l_{2}\right)}}{l_{1}+l_{2}+1} \cdots \frac{B_{l_{k-1}}^{\left(l_{k-1}\right)}}{l_{1}+\cdots+l_{k-1}+1} \frac{\xi^{l}}{l !} \\
= & \sum_{j=0}^{\infty} \sum_{l=0}^{j}\left(\begin{array}{l}
j \\
l
\end{array}\right) \sum_{l=0}^{\infty} 2^{l} \\
& \times \sum_{l_{1}+\cdots+l_{k-1}=l}^{l}\left(l_{1}, \ldots, l_{k-1}\right) \frac{B_{l_{1}}^{\left(l_{1}\right)}}{l_{1}+1} \frac{B_{l_{2}}^{\left(l_{2}\right)}}{l_{1}+l_{2}+1} \cdots \frac{B_{l_{k-1}}^{\left(l_{k-1}\right)}}{l_{1}+\cdots+l_{k-1}+1} C h_{j-l} \frac{\xi^{j !}}{j !}
\end{aligned}
$$

In view of (25) and (26), we complete the theorem.

$$
\begin{aligned}
\sum_{j=0}^{\infty} C h_{j}^{(k)}(\xi+\eta) \frac{z^{j}}{j !} & =\left(\frac{\operatorname{Ei}_{k}(\log (1+2 z))}{z(2+z)}\right)(1+z)^{\xi+\eta} \\
& =\left(\sum_{j=0}^{\infty} C h_{j}^{(k)}(\xi) \frac{z^{j}}{j !}\right)\left(\sum_{i=0}^{\infty}(\eta)_{i} \frac{z^{i}}{i !}\right) \\
& =\sum_{j=0}^{\infty}\left(\sum_{i=0}^{j}\left(\begin{array}{c}
j \\
i
\end{array}\right) C h_{j-i}^{(k)}(\xi)(\eta)_{i}\right) \frac{z^{j}}{j !}
\end{aligned}
$$$$
C h_{j}^{(2)}=\sum_{l=0}^{j}\left(\begin{array}{l}
j \\
l
\end{array}\right) \frac{2^{l} B_{l}^{l}}{l+1} C h_{j-l} .
$$

Theorem 3. For $j \geq 0$, we have

So, the proof is completed.

$$
C h_{j}^{(2)}(\xi+\eta)=\sum_{i=0}^{j}\left(\begin{array}{l}
j \\
i
\end{array}\right) C h_{j-i}^{(k)}(\xi)(\eta)_{i} .
$$

Proof. Considering (16), we have

Theorem 4. Let $j \geq 1$, then

$$
2 C h_{j}^{(k)}+j C h_{j-1}^{(k)}=2^{j+1} \sum_{i=1}^{j+1} \frac{1}{i^{k-1}} \frac{S_{1}(j+1, i)}{j+1} .
$$


Proof. Equation (16) can be written as

On the other hand, we have

$$
\begin{aligned}
\frac{\mathrm{Ei}_{k}(\log (1+2 z))}{z} & =(2+z) \sum_{j=0}^{\infty} C h_{j}^{(k)} \frac{z^{j}}{j !} \\
& =2 \sum_{j=0}^{\infty} C h_{j}^{(k)} \frac{z^{j}}{j !}+\sum_{j=0}^{\infty} C h_{j}^{(k)} \frac{z^{j+1}}{j !} \\
& =2 \sum_{j=0}^{\infty} C h_{j}^{(k)} \frac{z^{j}}{j !}+j \sum_{j=1}^{\infty} C h_{j-1}^{(k)} \frac{z^{j}}{j !} \\
& =\sum_{j=1}^{\infty}\left(2 C h_{j}^{(k)}+j C h_{j-1}^{(k)}\right) \frac{z^{j}}{j !} .
\end{aligned}
$$

$$
\begin{aligned}
\frac{\mathrm{Ei}_{k}(\log (1+2 z))}{z} & =\frac{1}{z} \sum_{i=1}^{\infty} \frac{(\log (1+2 z))^{i}}{(i-1) ! i^{k}} \\
& =\frac{1}{z} \sum_{i=1}^{\infty} \frac{(\log (1+2 z))^{i}}{(i-1) ! i^{k}}=\frac{1}{z} \sum_{i=1}^{\infty} \frac{1}{i^{k-1}} \frac{(\log (1+2 z))^{i}}{i !} \\
& =\frac{1}{z} \sum_{i=1}^{\infty} \frac{1}{i^{k-1}} \sum_{j=i}^{\infty} S_{1}(j, i) \frac{2^{j} z^{j}}{j !}=\frac{1}{z} \sum_{j=1}^{\infty} \sum_{i=1}^{j} \frac{1}{i^{k-1}} S_{1}(j, i) \frac{2^{j} z^{j}}{j !} \\
& =\sum_{j=1}^{\infty} \sum_{i=1}^{j+1} \frac{1}{i^{k-1}} \frac{S_{1}(j+1, i)}{j+1} 2^{j+1} \frac{z^{j}}{j !} .
\end{aligned}
$$

Therefore, by (31) and (32), we obtain the result.

Theorem 5. For $j \geq 0$, we have

$$
C h_{j}^{(k)}(1)+C h_{j}^{(k)}=\sum_{i=1}^{j+1} \frac{1}{i^{k-1}} \frac{S_{1}(j+1, i)}{j+1} 2^{j+1} .
$$

Proof. By (16), we see that

$$
\begin{aligned}
\sum_{j=1}^{\infty}\left[C h_{j}^{(k)}(1)+C h_{j}^{(k)}\right] \frac{z^{j}}{j !} & =\left(\frac{\operatorname{Ei}_{k}(\log (1+2 z))}{z}\right) \\
& =\sum_{j=1}^{\infty} \sum_{i=1}^{j+1} \frac{1}{i^{k-1}} \frac{S_{1}(j+1, i)}{j+1} 2^{j+1} \frac{z^{j}}{j !}
\end{aligned}
$$

which completes the proof of the theorem.

Theorem 6. Let $j$ be the nonnegative number. Then,

$$
C h_{j}^{(k)}(\xi)=\sum_{i=0}^{j} \sum_{r=0}^{i}\left(\begin{array}{l}
j \\
i
\end{array}\right)(\xi)_{r} S_{1}(i, r) C h_{j-i}^{(k)} .
$$

Proof. From (16), we note that

$$
\begin{aligned}
\sum_{j=0}^{\infty} C h_{j}^{(k)}(\xi) \frac{z^{j}}{j !} & =\left(\frac{\operatorname{Ei}_{k}(\log (1+2 z))}{z(2+z)}\right)\left(1+\log e^{z}\right)^{\xi} \\
& =\left(\sum_{j=0}^{\infty} C h_{j}^{(k)} \frac{z^{j}}{j !}\right)\left(\sum_{r=0}^{\infty}(\xi)_{r}\left(\log e^{z}\right)^{r}\right) \\
& =\left(\sum_{j=0}^{\infty} C h_{j}^{(k)} \frac{z^{j}}{j !}\right)\left(\sum_{i=0}^{\infty}\left(\sum_{r=0}^{i}(\xi)_{r} S_{1}(i, r)\right) \frac{z^{i}}{i !}\right) \\
& =\sum_{j=0}^{\infty}\left(\sum_{i=0}^{j} \sum_{r=0}^{i}\left(\begin{array}{c}
j \\
i
\end{array}\right)(\xi)_{r} S_{1}(i, r) C h_{j-i}^{(k)}\right) \frac{z^{j}}{j !} .
\end{aligned}
$$

By (16) and (36), we obtain the result.

Theorem 7. Let $j$ be the nonnegative number. Then,

$$
\sum_{r=0}^{j} C h_{j}^{(k)} S_{2}(j, r)=\sum_{p=0}^{j} \sum_{q=0}^{p}\left(\begin{array}{c}
p \\
q
\end{array}\right)\left(\begin{array}{c}
j \\
p
\end{array}\right) B_{p-q} E_{q} \frac{1}{(j-p+1)^{k}} \text {. }
$$

Proof. Replacing $z$ by $e^{z}-1$ in (16), we get 


$$
\begin{aligned}
\sum_{r=0}^{\infty} C h_{r}^{(k)} \frac{\left(e^{z}-1\right)^{r}}{r !} & =\frac{2 \mathrm{Ei}_{k}(z)}{\left(e^{z}-1\right)\left(e^{z}+1\right)} \\
\sum_{r=0}^{\infty} C h_{r}^{(k)} \sum_{j=r}^{\infty} S_{2}(j, r) \frac{z^{j}}{j !} & =\frac{2}{e^{z}+1} \frac{z}{e^{z}-1} \frac{\operatorname{Ei}_{k}(z)}{z} \\
\sum_{j=0}^{\infty}\left(\sum_{r=0}^{j} C h_{r}^{(k)} S_{2}(j, r)\right) \frac{z^{j}}{j !} & =\sum_{p=0}^{\infty} B \frac{z^{p}}{p !} \sum_{q=0}^{\infty} E_{q} \frac{z^{q}}{q !} \sum_{j=0}^{\infty} \frac{z^{j}}{j !(j+1)^{k}} \\
& =\sum_{p=0}^{\infty} \sum_{q=0}^{p}\left(\begin{array}{c}
p \\
q
\end{array}\right) B_{p-q} E_{q} \frac{z^{p}}{p !} \sum_{j=0}^{\infty} \frac{z^{j}}{j !(j+1)^{k}} \\
& =\sum_{j=0}^{\infty} \sum_{p=0}^{j} \sum_{q=0}^{p}\left(\begin{array}{c}
p \\
q
\end{array}\right)\left(\begin{array}{c}
n \\
p
\end{array}\right) B_{p-q} E_{q} \frac{1}{(j-p+1)^{k}} \frac{z^{j}}{j !} .
\end{aligned}
$$

Therefore, by (38) and (39), we get the result.

Theorem 8. Let $j$ be nonnegative number. Then,

$$
C h_{j}^{(k)}(\xi)=\sum_{s=0}^{j-i}\left(\begin{array}{l}
j \\
i
\end{array}\right) E_{s}(\xi) S_{1}(j-i, s) \sum_{r=0}^{i} \frac{1}{(r+1)^{k-1}} 2^{i} \frac{S_{1}(i+1, r+1)}{i+1}
$$

Proof. From (16), we have

$$
\begin{aligned}
& \sum_{j=0}^{\infty} C h_{j}^{(k)}(\xi) \frac{z^{j}}{j !}=\frac{\operatorname{Ei}_{k}(\log (1+2 z))}{z(2+z)}(1+z)^{\xi} \\
& =\frac{1}{z(z+2)}(1+z)^{\xi} \operatorname{Ei}_{k}(\log (1+2 z))=\frac{1}{z(z+2)}(1+z)^{\xi} \sum_{r=1}^{\infty} \frac{(\log (1+2 z))^{r}}{(r-1) ! r^{k}} \\
& =\frac{1}{z(z+2)}(1+z)^{\xi} \sum_{r=0}^{\infty} \frac{(\log (1+2 z))^{r+1}}{r !(r+1)^{k}} \\
& =\frac{1}{z(z+2)}(1+z)^{\xi} \sum_{r=0}^{\infty} \frac{1}{(r+1)^{k-1}} \frac{1}{(r+1) !}(\log (1+2 z))^{r+1} \\
& =\frac{2}{z+2}(1+z)^{\xi} \sum_{i=0}^{\infty} \sum_{r=0}^{i} \frac{1}{(r+1)^{k-1}} 2^{i} \frac{S_{1}(i+1, r+1)}{i+1} \frac{z^{i}}{i !} \\
& =\frac{2}{e^{\log (1+z)}+1} e^{\xi \log (1+z)} \sum_{l=0}^{\infty} \sum_{r=0}^{i} \frac{1}{(r+1)^{k-1}} 2^{i} \frac{S_{1}(i+1, r+1)}{i+1} \frac{z^{i}}{i !} \\
& =\left(\sum_{s=0}^{\infty} E_{s}(\xi) \frac{1}{s !}(\log (1+z))^{s}\right)\left(\sum_{i=0}^{\infty} \sum_{r=0}^{i} \frac{1}{(r+1)^{k-1}} 2^{i} \frac{S_{1}(i+1, r+1)}{i+1} \frac{z^{i}}{i !}\right) \\
& =\left(\sum_{j=0}^{\infty} \sum_{s=0}^{j} E_{s}(\xi) S_{1}(j, s) \frac{z^{j}}{j !}\right)\left(\sum_{i=0}^{\infty} \sum_{r=0}^{i} \frac{1}{(r+1)^{k-1}} 2^{i} \frac{S_{1}(i+1, r+1)}{i+1} \frac{z^{i}}{i !}\right) \\
& =\sum_{j=0}^{\infty}\left(\sum_{s=0}^{j-i}\left(\begin{array}{l}
j \\
i
\end{array}\right) E_{s}(\xi) S_{1}(j-i, s) \sum_{r=0}^{i} \frac{1}{(r+1)^{k-1}} 2^{i} \frac{S_{1}(i+1, r+1)}{i+1}\right) \frac{z^{j}}{j !} .
\end{aligned}
$$


Thus, by (41) and (42), we complete the proof.

\section{Type 2 Unipoly-Changhee Numbers and Polynomials}

Recently, Kim and Kim [12] introduced the unipoly function $u_{k}(\xi \mid p)$ by

$$
u_{k}(\xi \mid p)=\sum_{j=1}^{\infty} \frac{p(j)}{j^{k}} \xi^{j}, \quad(k \in \mathbb{Z}),
$$

where $u_{k}(\xi \mid p)$ is attached to polynomials $p(\xi)$ and $p$ is any arithmetic function which is real or complex.

Putting $k=1,(43)$ to get (see [5])

$$
u_{k}(x \mid 1)=\sum_{j=1}^{\infty} \frac{\xi^{j}}{j^{k}}=\operatorname{Li}_{k}(\xi)
$$

and it is called the polylogarithm function.

By using (7) and (43), we consider the unipoly-Changhee polynomials attached to polynomials $p(\xi)$ by

$$
\frac{1}{z(2+z)} u_{k}(\log (1+2 z) \mid p)(1+z)^{\xi}=\sum_{j=0}^{\infty} C h_{j, p}^{(k)}(\xi) \frac{z^{j}}{j !} .
$$

When $\xi=0, C h_{j, p}^{(k)}=C h_{j, p}^{(k)}(0)$ are called the unipolyChanghee numbers attached to $p$.

Theorem 9. Let $j$ be the nonnegative number. Then,

$$
C h_{j,(1 / \Gamma)}^{(k)}=C h_{j}^{(k)} \text {. }
$$

Proof. Taking $p(j)=1 / \Gamma(j)$ in (45), we have

$$
\begin{aligned}
\sum_{j=0}^{\infty} C h_{j,(1 / \Gamma)}^{(k)} \frac{z^{j}}{j !} & =\frac{1}{z(2+z)}(1+z) u_{k}\left(\log (1+2 z) \mid \frac{1}{\Gamma}\right) \\
& =\frac{1}{z(2+z)} \sum_{r=1}^{\infty} \frac{(\log (1+2 z))^{r}}{r^{k}(r-1) !} \\
& =\frac{1}{z(2+z)} \operatorname{Ei}_{k}(\log (1+2 z))=\sum_{j=0}^{\infty} C h_{j}^{(k)} \frac{z^{j}}{j !}
\end{aligned}
$$

Thus, we complete the proof.

Theorem 10. For $j \geq 0$ and $k \in \mathbb{Z}$, we have

$$
C h_{j, p}^{(k)}(\xi)=\sum_{i=0}^{j} \sum_{r=0}^{i}\left(\begin{array}{l}
j \\
i
\end{array}\right) \frac{p(r+1)(r+1) !}{(r+1)^{k}} \frac{S_{1, \lambda}(i+1, r+1) 2^{i}}{i+1} C h_{j-i}(\xi) .
$$

Proof. From (45), we have

$$
\begin{aligned}
\sum_{j=0}^{\infty} C h_{j, p}^{(k)}(\xi) \frac{z^{j}}{j !} & =\frac{1}{z(2+z)}(1+z)^{\xi} u_{k}(\log (1+2 z) \mid p) \\
& =\frac{1}{z(2+z)}(1+z)^{\xi} \sum_{r=1}^{\infty} \frac{p(r)}{r^{k}}(\log (1+2 z))^{r} \\
& =\frac{1}{z(2+z)}(1+z)^{\xi} \sum_{r=0}^{\infty} \frac{p(r+1)}{(r+1)^{k}}(\log (1+2 z))^{r+1} \\
& =\frac{1}{z(2+z)}(1+z)^{\xi} \sum_{r=0}^{\infty} \frac{p(r+1)(r+1) !}{(r+1)^{k}} \sum_{i=r+1}^{\infty} S_{1}(i, r+1) \frac{2^{i} z^{i}}{i !} \\
& =\frac{2}{2+z}(1+z)^{\xi} \sum_{r=0}^{\infty} \frac{p(r+1)(r+1) !}{(r+1)^{k}} \sum_{i=r}^{\infty} \frac{S_{1}(i+1, r+1) 2^{i}}{i+1} \frac{z^{i}}{i !} \\
& =\sum_{j=0}^{\infty} C h_{j}(\xi) \frac{z^{j}}{j !} \sum_{i=0}^{\infty}\left(\sum_{r=0}^{i} \frac{p(r+1)(r+1) !}{(r+1)^{k}} \frac{S_{1}(i+1, r+1) 2^{i}}{i+1}\right) \frac{z^{i}}{i !} \\
& =\sum_{j=0}^{\infty}\left(\sum_{i=0}^{j} \sum_{r=0}^{i}\left({ }_{j}^{i}\right) \frac{p(r+1)(r+1) !}{(r+1)^{k}} \frac{S_{1}(i+1, r+1) 2^{i}}{i+1} C h_{j-i}(\xi)\right) \frac{z^{j}}{j !}
\end{aligned}
$$


So, the proof is completed.

Corollary 3. Let $j$ be the nonnegative number. Then,

$$
C h_{j,(1 / \Gamma)}^{(k)}(\xi)=\sum_{i=0}^{j} \sum_{r=0}^{i}\left(\begin{array}{l}
j \\
i
\end{array}\right) \frac{1}{(r+1)^{k-1}} \frac{S_{1}(i+1, r+1) 2^{i}}{i+1} C h_{j-i}(\xi) .
$$

Theorem 11. Let $j$ be the nonnegative number. Then,

$$
C h_{j, p}^{(k)}(\xi)=\sum_{r=0}^{j}\left(\begin{array}{l}
j \\
r
\end{array}\right) C h_{r, p}^{(k)}(\xi)_{j-r} .
$$

Proof. Using (45), we have

$$
\begin{aligned}
\sum_{j=0}^{\infty} C h_{j, p}^{(k)}(\xi) \frac{z^{j}}{j !} & =\frac{1}{z(2+z)} u_{k}(\log (1+2 z) \mid p)(1+z)^{\xi} \\
& =\frac{1}{z(2+z)} u_{k}(\log (1+2 z) \mid p) \sum_{j=0}^{\infty}(\xi)_{j} \frac{z^{j}}{j !} \\
& =\sum_{r=0}^{\infty} C h_{r, p}^{(k)} \frac{z^{r}}{r !} \sum_{j=0}^{\infty}(\xi) \frac{z^{j}}{j !} \\
& =\sum_{j=0}^{\infty}\left(\sum_{r=0}^{j}\left(\begin{array}{l}
j \\
r
\end{array}\right) C h_{r, p}^{(k)}(\xi)_{j-r}\right) \frac{z^{j}}{j !} .
\end{aligned}
$$

$$
\begin{aligned}
& \sum_{j=0}^{\infty} C h_{j, p}^{(k)} \frac{z^{j}}{j !}=\frac{1}{z(2+z)} u_{k}(\log (1+2 z) \mid p) \\
& =\frac{1}{z(2+z)} \sum_{s=0}^{\infty} \frac{p(s+1)}{(s+1)^{k}}(\log (1+2 z))^{s+1} \\
& =\frac{\log (1+2 z)}{z(2+z)} \sum_{s=0}^{\infty} \frac{p(s+1) s !}{(s+1)^{k}} \sum_{l=s}^{\infty} S_{1}(l, m) \frac{2^{l} z^{l}}{l !} \\
& =\frac{\log (1+2 z)}{2 z} \frac{2}{2+z} \sum_{m=0}^{\infty} \frac{p(m+1) m !}{(m+1)^{k}} \sum_{l=m}^{\infty} S_{1}(l, m) 2 \frac{z^{z^{l}}}{l !} \\
& =\sum_{r=0}^{\infty} D_{r} \frac{2^{r} z^{r}}{r !} \sum_{j=0}^{\infty} C h_{j} \frac{z^{j}}{j !} \sum_{i=0}^{\infty}\left(\sum_{m=0}^{i} \frac{p(m+1) m !}{(m+1)^{k}} S_{1}(i, m) 2^{i}\right) \frac{z^{i}}{i !} \\
& =\sum_{n=0}^{\infty} \sum_{r=0}^{n}\left(\begin{array}{c}
n \\
r
\end{array}\right) 2^{r} D_{r} C h_{n-r} \frac{t^{n}}{n !} \sum_{l=0}^{\infty}\left(\sum_{m=0}^{l} \frac{p(m+1) m !}{(m+1)^{k}} S_{1}(l, m) 2^{l}\right) \frac{t^{l}}{l !} \\
& =\sum_{j=0}^{\infty} \sum_{r=0}^{j-i} \sum_{i=0}^{j}\left(\begin{array}{c}
j-i \\
r
\end{array}\right)\left(\begin{array}{c}
j \\
i
\end{array}\right) 2^{i+r} D_{r} C h_{j-r-i} \sum_{s=0}^{i} \frac{p(s+1) s !}{(s+1)^{k}} S_{1}(i, s) \frac{z^{j}}{j !} .
\end{aligned}
$$


Therefore, by (45) and (54), we obtain the result.

\section{Conclusion}

In the previous sections, we have touched on the problem of recognizing the algebraic structure underlying the polyChanghee polynomials as given by definition (16). The analysis is aimed at accounting for the wealth of the properties exhibited by these polynomials within the context of the poly-Changhee numbers and polynomials which provide a unifying formalism where the theory of special functions can be framed inherently. Some analogies with the theory of poly-Changhee numbers and polynomials can be recognized and usefully exploited to infer further properties of these polynomials and links with other special functions. Let us stress that the scheme suggested by the following properties of Changhee numbers and polynomials $\mathrm{Ch}_{n}(x)$ studied in detail by Kim and Kim [14] can be applied to connect other special functions of relevance in mathematical physics.

\section{Data Availability}

No data were used to support this study.

\section{Conflicts of Interest}

The authors declare that they have no conflicts of interest.

\section{Authors' Contributions}

All authors contributed equally to the manuscript and typed, read, and approved final manuscript.

\section{References}

[1] W. A. Khan, M. Acikgoz, and U. Duran, "Note on the type 2 degenerate multi-poly-Euler polynomials," Symmetry, vol. 12, Article ID 1691, 2020.

[2] D. V. Dolgy, G.-W. Jang, H.-I. Kwon, and T. Kim, "A note on Carlitz's type q-Changhee numbers and polynomials," 2017, https://arxiv.org/abs/1708.06547v1.

[3] D. S. Kim and T. Kim, "Higher-order Changhee numbers and polynomials," 2013, https://arxiv.org/abs/1310.7103v1.

[4] J. S. So and Y. Simsek, "Derivation of computational formulas for Changhee polynomials and their functional and differential equations," Journal of Inequalities and Applications, vol. 2020, no. 1, p. 149, 2020.

[5] M. Kaneko, "Poly-Bernoulli numbers," Journal de Théorie des Nombres de Bordeaux, vol. 9, no. 1, pp. 221-228, 1997.

[6] W. A. Khan and H. Haroon, "Some symmetric identities for the generalized Bernoulli, Euler and Genocchi polynomials associated with Hermite polynomials," SpringerPlus, vol. 5, no. 1, p. 1920, 2016.

[7] W. A. Khan, K. S. Nisar, U. Duran, M. Acikgoz, and S. Araci, "Multifarious implicit summation formulae of Hermite-based poly-Daehee polynomials," Applied Mathematics and Information Sciences, vol. 12, no. 2, pp. 305-310, 2018.

[8] W. A. Khan, G. Muhiuddin, A. Muhyi, and D. Al-Kadi, "Analytical properties of type 2 degenerate poly-Bernoulli polynomials associated with their applications," Advances in Difference Equations, vol. 2021, pp. 1-18, 2021.
[9] W. A. Khan, A. Muhyi, R. Ali, K. A. H. Alzobydi, M. Singh, and P. Agarwal, "A new family of degenerate poly-Bernoulli polynomials of the second kind with its certain related properties," AIMS Mathematics, vol. 6, no. 11, pp. 12680-12697, 2021.

[10] W. A. Khan, R. Ali, K. A. H. Alzobydi, and N. Ahmed, "A new family of degenerate poly-Genocchi polynomials with its certain properties," Journal of Function Spaces, vol. 2021, Article ID 6660517, 8 pages, 2021.

[11] D. S. Kim and T. Kim, "Higher-order Bernoulli and polyBernoulli mixed type polynomials," Georgian Mathematical Journal, vol. 22, pp. 265-272, 2015.

[12] D. S. Kim and T. Kim, "A note on polyexponential and unipoly functions," Russian Journal of Mathematical Physics, vol. 26, no. 1, pp. 40-49, 2019.

[13] D. S. Kim, T. Kim, and J. Seo, "A note on Changhee polynomials and numbers," Advanced Studies in Theoretical Physics, vol. 7, no. 1, pp. 993-1003, 2013.

[14] T. Kim and D. S. Kim, "A note on nonlinear Changhee differential equations," Russian Journal of Mathematical Physics, vol. 23, no. 1, pp. 88-92, 2016.

[15] G.-W. Jang, D. S. Kim, and T. Kim, "Degenerate Changhee numbers and polynomials of the second kind," Advanced Studies in Contemporary Mathematics, vol. 27, no. 4, pp. 609-624, 2017.

[16] D. S. Kim and T. Kim, "A note on type 2 Changhee and Daehee polynomials," Revista de la Real Academia de Ciencias Exactas, Físicas y Naturales, Serie A Matemáticas, vol. 113, no. 3, pp. 2783-2791, 2019.

[17] G. Muhiuddin, W. A. Khan, U. Duran, and D. Al-Kadi, "Some identities of the multi poly-Bernoulli polynomials of complex variable," Journal of Function Spaces, vol. 2021, Article ID 7172054, 8 pages, 2021

[18] G. Muhiuddin, W. A. Khan, and U. Duran, "Two variable type 2 Fubini polynomials," Mathematics, vol. 9, no. 281, pp. 1-13, 2021.

[19] G. Muhiuddin, W. Khan, A. Muhyi, and D. Al-Kadi, "Some results on type 2 degenerate poly-Fubini polynomials and numbers," Computer Modeling in Engineering and Sciences, vol. 129, no. 2, pp. 1051-1073, 2021.

[20] G. Muhiuddin, W. A. Khan, and D. Al-Kadi, "Construction on the degenerate poly-Frobenius-Euler polynomials of complex variable," Journal of Function Spaces, vol. 2021, Article ID 3115424, 9 pages, 2021.

[21] L. Lewin, Polylogarithms and Associated Functions; with a Foreword by A.J. Vander Poorten, North-Holland Publishing, Amsterdam, NY, USA, 1981.

[22] D. S. Lee, H. K. Kim, and L. C. Jang, "Type 2 degenerate polyEuler polynomials," Symmetry, vol. 12, Article ID 1011, 2020. 